\title{
Uso de medicamentos fitoterápicos como opção anti-inflamátoria na odontologia
}

\author{
Use of phytotherapy medicines as anti- \\ inflammatory option in dentistry
}

\section{Vivian Lopes Moreira' ${ }^{1}$ \\ Andréa Padre Peixoto ${ }^{2}$ (1) Antônio Marcio Teixeira Marchionni3 ${ }^{3}$}

\begin{abstract}
${ }^{1}$ Autora para correspondência. Escola Bahiana de Medicina e Saúde Pública (Salvador). Bahia, Brasil. vivianlopes1998@gmail.com ${ }^{2}$ Centro de Especialização em Homeopatia de Londrina (Londrina). Paraná, Brasil. andreapadre@gmail.com ${ }^{3}$ Escola Bahiana de Medicina e Saúde Pública (Salvador). Bahia, Brasil. amtmarchionni@bahiana.edu.br
\end{abstract}

\begin{abstract}
RESUMO | O termo fitoterapia vem do latim, "phyton" que significa planta, e "terapia" relacionado ao tratamento, cujo primeiro relato manuscrito em 1500 a.C. titulado "Papiro de Ebers". A fitoterapia é a ciência que utiliza plantas para o tratamento de diversas enfermidades humanas. No Brasil, estudos demonstram um interesse do Sistema Único de Saúde (SUS) a respeito dos medicamentos fitoterápicos entre as décadas de 70 e 80 , principalmente na atenção primária à saúde devido a diminuição do custo, porém poucos estudos observacionais são encontrados na literatura a respeito da utilização dos mesmos no sistema. O presente estudo trata-se de uma revisão de literatura com o objetivo de relatar os principais medicamentos fitoterápicos com ação antiinflamatória, como uma opção terapêutica para pacientes que não podem ou não desejam fazer uso dos antiinflamatórios não-esteroides (AINES). Para a sua construção realizou-se uma busca por artigos nas bases de dados PubMed, Scielo e na plataforma BVSalud, com linha do tempo estabelecida de 2009 a 2020. Pôde se concluir, que os medicamentos fitoterápicos podem ser utilizados como uma alternativa terapêutica segura em substituição dos AINES, por oferecer mecanismo de ação semelhante e efeitos colaterais reduzidos ou até mesmo nulo. Podendo assim se tornar realidade na prática odontológica por Cirurgiões-Dentistas.
\end{abstract}

PALAVRAS-CHAVE: Fitoterapia. Medicamentos fitoterápicos. Odontologia. Anti-inflamatórios.
ABSTRACT | The term phytotherapy comes from the Latin, "phyton" which means plant, and "therapy" related to treatment, whose first handwritten account in 1500 BC entitled "Papers of Ebers". Phytotherapy is the science that uses plants for the treatment of various human diseases. In Brazil, studies show an interest of the Unified Health System (SUS) regarding herbal medicines between the 70s and 80s, mainly in primary health care due to the cost reduction, however few observational studies are found in the literature regarding their use in the system. The present study is a literature review in order to report the main herbal medicines with anti-inflammatory action, as a therapeutic option for patients who cannot or do not wish to use non-steroidal antiinflammatory drugs (NSAIDs). For its construction, a search was performed for articles in the PubMed, Scielo and BVSalud databases, with a timeline established from 2009 to 2020. It was concluded that herbal medicines can be used as a safe therapeutic alternative in replacement of NSAIDs, as it offers a similar mechanism of action and reduced or even zero side effects. Thus, it can become a reality in dental practice by Dental Surgeons.

KEYWORDS: Phytotherapy. Phytotherapeutic Drugs. Dentistry. Inflammation. 


\section{Introdução}

O termo fitoterapia vem do latim, "phyton" que significa planta, mais o termo "terapia" relaciona-se ao tratamento. É um termo antigo, com o primeiro relato manuscrito em 1500 a.C. titulado "Papiro de Ebers". A fitoterapia é conceituada como a ciência atuante na utilização de plantas como benefício a raça humana na diminuição de sintomas e tratamento de doenças ${ }^{1,2}$.

Desde o primeiro relato mencionado na história, a fitoterapia apresentou-se como um recurso na qual a própria natureza poderia nos beneficiar e sua utilização foi vigorosa até meados do século $X X$, quando a revolução industrial e seus avanços trouxeram tecnologias e o desenvolvimento dos medicamentos alopáticos ${ }^{1}$.

Entre a década de 70 e 80, a Organização Mundial da Saúde (OMS) passou a incentivar estudos e testes de plantas medicinais. Com isso, o Sistema Único de Saúde (SUS) do Brasil, possibilitou o uso de medicamentos fitoterápicos na atenção primária à saúde, devido ao custo acessível e grande disponibilidade em matéria prima ${ }^{23}$.

No ano de 2006, implantou-se as Práticas Integrativas e Complementares (PNPIC), que inclui a fitoterapia como opção preventiva e terapêutica para os usuários do SUS'. A indústria de fitoterápicas está em franco desenvolvimento no mundo e pode representar uma oportunidade ao setor farmacêutico no Brasil, esta não é apenas pela abundante biodiversidade brasileira, mas pelo tradicional e científico conhecimento acumulado sobre as plantas medicinais ${ }^{4}$.

Além disso, pelo harmônico momento de humanização da relação profissional/paciente, em políticas públicas. Existe uma excelente aceitação popular devido ao conhecimento dos efeitos terapêuticos das plantas e reações adversas mínimas $2,5$.

Dentre as desvantagens dos fitoterápicos destacamse os danos à saúde quando administrados com outras drogas e de forma equivocada, semelhantemente com os alopáticos. Temos como exemplo de efeitos colaterais dos medicamentos fitoterápicos: inflamações na pele, reações subcutâneas, náuseas, vômitos, pruridos e outros. Sendo assim, é importante conhecer seus princípios ativos e aspectos relacionados para prescrevê-los com segurança ${ }^{1,6}$.
De acordo com a Resolução CFO 82/2008, no Capítulo II, Art. 7\%:

"A Fitoterapia na Odontologia se destina aos estudos dos princípios científicos da fitoterapia e plantas medicinais embasados nas multidisciplinaridades inseridas na pratica profissional, o resgate do saber popular e no uso e aplicabilidade desta terapêtica na Odontologia. Respeitando o limite de atuação do campo profissional do cirurgião-dentista ${ }^{\text {?." }}$

A resolução afirma que "O avanço das políticas públicas de incremento às práticas integrativas e complementares nas ciências da saúde cria novas perspectivas de mercado de trabalho para o cirurgião-dentista ${ }^{7} . "$

No art. $1^{\circ}$ desta resolução diz "reconhecer o exercício pelo cirurgião-dentista das seguintes práticas integrativas e complementares à saúde bucal: Acupuntura, Fitoterapia, Terapia Floral, Hipnose, Homeopatia e Laserterapia" No art. 2 "Será considerado habilitado pelos Conselho Federal e Regional de odontologia, para as práticas definidas no artigo anterior, o cirurgião-dentista que atender aos dispostos nessa resolução? ${ }^{7}$

A odontologia utiliza bastante e frequentemente antiinflamatórios em sua pratica clínica. Estes medicamentos possuem diversos efeitos colaterais sobre o sistema digestório, sobre os rins e plaquetas. E o número de pacientes impossibilitados de fazer uso destes medicamentos é cada vez maior, devido a alergias e preexistentes doenças renais, hepáticas entre outras. Dessa forma, é importante ter o auxílio de medicamentos fitoterápicos que possuam atividades antiinflamatória e menor agressão sobre o organismo.

O presente trabalho é necessário para que se possa analisar a eficácia e mecanismo de ação dos medicamentos fitoterápicos a serem utilizados na Odontologia. O objetivo desta revisão de literatura é relatar os principais medicamentos e suas ações antiinflamatórias, para que a utilização destes se intensifique na prática odontológica, e possam representar uma opção terapêutica para pacientes que não podem ou não desejam fazer uso dos antiinflamatórios não esteroides (AINES). 


\section{Metodologia}

O presente trabalho compreende como uma revisão de literatura que consistiu em um levantamento bibliográfica envolvendo artigos científicos encontrados nos bancos de dados: BVSalud, Pubmed e Scielo.

As palavras-chaves utilizadas como estratégia de busca, foram: "fitoterapia" (phytotherapy), "odontologia" (dentistry), "medicamentos fitoterápicos" (herbal medicines), "farmacocinética" (phamacokinetics), inflamação (inflammation). Na plataforma BVSalud foram encontradas 51 referências, no Scielo 15 referências, no PubMed 05 referências, porém utilizou-se 47 referências para a resolução. No momento da seleção dos artigos foi realizada a leitura do resumo para verificar os que apresentavam características dos padrões de seleção do estudo.

Como critério de inclusão preconizou-se uma linha de tempo entre 2009 a 2020, com seleção de artigos no idioma inglês, português e espanhol, dentre esses seis artigos anteriores a este período foram anexados, referente a importância das informações ofertada por eles. Além de artigos, o livro Farmacologia Ilustrada $5^{a}$ edição, e o Formulário de fitoterápicos da farmacopeia brasileira, foram utilizados como referência para o estudo. Os critérios de exclusão deste trabalho foram os artigos que não abordavam sobre a inflamação e não faziam parte da linha do tempo estabelecida para a construção dessa revisão.

\section{Revisão de literatura}

\section{Mecanismo de ação aines}

Para elucidar a eficácia dos medicamentos fitoterápicos como opção antiinflamatória na Odontologia, foi necessário estudar o mecanismo de ação dos AINES, com finalidade de encontrar alguma semeIhança entre eles.

Os principais antiinflamatórios prescritos na Odontologia são os AINES, estes se caracterizam por sua atividade antipirética, anti-inflamatória e analgésica. Principalmente utilizados em urgência endodôntica, cirurgias e etc $\stackrel{8}{\text {. }}$
O mecanismo de ação desses medicamentos atua principalmente inibindo as enzimas Ciclo-oxigenase (COX), e consequentemente reduzindo da conversão do ácido araquidônico em prostaglandinas, com efeitos desejados e indesejados ${ }^{8,9}$.

Cada categoria dos AINES possui um mecanismo de ação, são esses: os salicilatos agem fazendo o bloqueio das sínteses de prostaglandinas no hipotálamo e nos sítios-alvo da periferia; ácido propiônico que agem inibindo a síntese das prostaglandinas porém não os leucotrienos; ácido acético age inibindo a ciclo-oxigenase; oxicam inibe a COX-1 e a COX-2, porém se direciona mais para a COX-1; acetaminofeno inibe a síntese de prostaglandinas no sistema nervo-

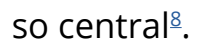

Os AINES apresentam nefrotoxicidade renal significativas que foram estudadas nos últimos tempos, os espectros mais estudados são: necrose tubular aguda, insuficiência renal crônica, hipertensão renal crônica, entre outros ${ }^{10}$.

O acetominofeno é um AINE que traz um risco aos indivíduos acerca da sua hepatotoxicidade. A ANVISA solicita que os profissionais não utilizem este medicamento em doses acima de $325 \mathrm{~g}$ pois não há comprovação de benefícios que compensem a risco de ocorrência de lesão hepática ${ }^{11}$.

Diversos estudos afirmaram que a nefrotoxicidade dos AINES é dada pela ação inibitória das sínteses de prostaglandinas através das cicloxigenases ${ }^{10,12}$. Lucas et al. $\frac{13}{}$ afirmaram que a maioria dos efeitos colaterais é relacionado com a inibição da COX-1 devido a ação de limpeza celular, e que os efeitos associados a COX2 se dá pela manutenção hidroeletrolítica no âmbito renal que causa danos em situações de desidratação. Batlouni ${ }^{14}$ afirmou que "as evidências sobre o aumento do risco cardiovascular com o uso de AINES, particularmente dos inibidores seletivos da COX-2, são ainda incompletas."

Por estes motivos a utilização dos AINES em pacientes renais crônicos e com doenças cardiovasculares se encontra em grupos de risco, principalmente por estes induzirem alterações deletérias ${ }^{10}$. 


\section{Medicamentos fitoterápicos com efeito anti- inflamatório mais utilizados na odontologia}

\section{Própolis}

A Própolis é uma resina elaborada por abelhas da espécie "Apis Mellifera". Estas abelhas coletam substâncias de rachaduras na casca das árvores, e outras partes da planta. No Brasil, a própolis é comumente encontrada na região Sudeste $\mathrm{z}^{2,15}$.

A composição fitoquímica da Própolis brasileira representa a biodiversidade do próprio Brasil, possuindo pelo menos 13 diferentes tipos de Própolis, que possuem compostos como apigenina, artepilina $C$, vestitol, neovestitol, entre outros que são moléculas que possuem papel significativo no processo inflamatório ${ }^{16}$.

As propriedades terapêuticas são antimicrobianas, antiinflamatória e antisséptica. Na Odontologia é normalmente disponibilizada em forma de pasta de dente, pastilhas, pó, colutórios e extratos².

Na Odontologia, a Própolis mostrou-se beneficente nos pacientes que estão em tratamento de quimioterapia e radioterapia. Salehi et al. ${ }^{17}$ afirmaram que a prescrição de própolis oral melhora a saúde bucal dos pacientes e reduz possíveis efeitos colaterais da mucosite oral, incluindo as úlceras orais e distúrbios alimen-

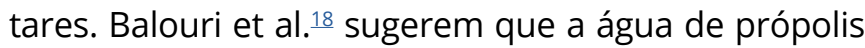
enxaguatório bucal é seguro e eficaz na prevenção e tratamento para mucosite induzida por radioterapia.

Aleluia et al. 2 e Parolia et al. $\frac{15}{}$ afirmaram que os flavonoides e ácido cafeico presentes na Própolis desempenham o papel na redução da resposta inflamatória, inibindo ácido araquidônico e a síntese de prostaglandina, além disso, ativam o timo e auxiliam o sistema imunitário através da atividade fagocítica. Por esse motivo, esta é indicada na terapêutica pósoperatória, capeamento pulpar direto e indutor de formação de dentina reparadora.

Parolia et al. $\frac{15}{15}$ afirmaram que a Própolis possui outro mecanismo de ação na diminuição da produção do nitrito, possuindo uma resposta anti-inflamatória. Desfrutando assim de uma ação mais ampla, pois, além de apresentar diminuição de prostaglandinas, age também na redução do oxido nítrico e aumenta a atividade fagocitária.
Franchin et al. $\frac{16}{}$ discutiram sobre o papel imunomodulador da Própolis através da inibição de citocinas inflamatórias tal como TNF-a (alfa) e quimiocinas, além de inibir a NF-kappaßeta e inibição dos neutrófilos. Afirmando que o mecanismo de ação da Própolis não deixa a desejar em comparação com os AINES, e não obtêm o efeito colateral que os mesmos trazem.

Stähli et al. 19 apresentaram um componente central da Própolis chamado de éster fenetílico do ácido cafeico (CAPE), este componente exerce efeito através da indução de heme oxigenase 1 (HO1) e por isto possui ação antioxidante, e mecanismo de ação antiinflamatória através da inibição de NF-kß. Afirmando que a aplicação da Própolis melhora o status periodontal em pacientes diabético, considerando que a doença periodontal é causada por inflamação crônica e danos oxidativos.

Segundo autores, os pacientes que apresentem sensibilidade ao ácido cafeico e ao polén possui hipersensibilidade a Própolis, dentre esses sintomas temos: erupções cutâneas, inchaço, coceira ou feridas na boca? .

\section{Myracrodruon urundeuva}

A Myracrodruon urundeuva é uma planta medicinal, mais conhecida como aroeira, e originada de uma árvore nativa da América do Sul. No Brasil, essa espécie se encontra nas regiões nordeste e sudeste, lugares secos do cerrado. A mesma possui outros nomes populares, tal como: aroeira-do-sertão, aroeira-da-serra, urindeúva e arindéuva ${ }^{20}$.

A aroeira normalmente é apresentada em formato de gel e óvulo, que contém ação cicatrizante, anti-inflamatória, antisséptica e para uso ginecológico ${ }^{21,22}$.

Os residentes destas regiões onde a aroeira é encontrada, indicam como anti-inflamatório e cicatrizante no tratamento de feridas, para gastrite, úlceras e outros ${ }^{10}$.

Soares et al. ${ }^{22}$ determinaram a atividade antimicrobiana in vitro, sobre a linhagem padrão Streptococcus mutans (ATCC 2575) através da técnica de infusão em ágar para definir a diluição inibitória máxima. Concluiu-se que a tintura da aroeira a $20 \%$ apresentou atividade antibacteriana in vitro sobre $S$. mutans e foi eficaz na redução da contaminação de escovas dentais. Machado et al. $\underline{23}$ avaliaram o efeito do extrato etanólico da aroeira na viabilidade do fibroblasto gengival, para isso eles 
banharam os fibroblastos em uma placa com 96 poços e incubaram por $24 \mathrm{~h}$, dessa maneira concluíram que a aroeira etanólica, promoveu uma mudança na viabilidade do fibroblasto gengival.

Souza et al. ${ }^{24}$ afirmaram a eficácia da aroeira como uso anti-inflamatório e antiulcerante, porém sem demonstrar o mecanismo de ação da planta nesse processo.

A literatura não apresenta estudos que afirmam o mecanismo de ação da aroeira como um medicamento anti-inflamatório, porém alguns estudos relatam sobre a sua eficácia antibacteriana.

\section{Arnica montana}

A Arnica montana é uma planta fitoterápica normalmente encontrada em regiões montanhosas, tal como: Europa e no Norte da Ásia ${ }^{6,25}$. Pertence à família das Aesteraceae que inclui: Arnica montana, Arnica chamissonis, Arnica fulgens, Arnica cordifolia e Arnica sororia ${ }^{26}$.

A forma de utilização mais comum da arnica é por infusão (3g em $150 \mathrm{~mL}$ na xicara de chá), por compressa aplicada no local 2 a $3 x$ ao dia, uso adulto e infantil. A utilização da tintura Arnica montana via oral pode causar gastroenterites, distúrbios cardiovasculares, falta de ar e morte. Há outras contraindicações como: não aplicar em feridas abertas, evitar o uso em pessoas que tenham sensibilidade as plantas da família Aesteraceae, e não utilizar num período maior que 07 dias $^{21,27}$.

Na Odontologia a Arnica montana é muito utilizada para redução de edema, dor e atividade anti-inflamatória. Quem mais utiliza desses medicamentos são os cirurgiões bucomaxilofacial em exodontia de terceiros molares, após traumatismos de face ${ }^{25}$.

Ianniti et al. $\underline{26}$ Berges et al. $\underline{28}$ Tornhamre et al. $\underline{29}$ afirmaram que há uma substância dentro da arnica conhecida como Helanina, que é responsável pelo mecanismo de ação da arnica montana através da diminuição do complexo Kappa B (NFkB) em células T e B, e a expressão dos receptores de superfície celular CD25, CD28, CD27 e CD 10b que desempenham papel fundamental na ativação de NFkB em células T.

Verma et al..$^{30}$ afirmaram que o mecanismo de ação da arnica vai da inibição de óxido nítrico (NO), da cicloxigenase-2 (COX-2) até o fator de necrose tumoral (TNF-a). Jäger et al. ${ }^{31}$ já destacaram que a ação da arnica pode ser devido às propriedades anti-inflamatórias, redução da biossíntese de TNF-a e IL-1 $\beta$ que são mediadores pro-inflamatórios.

Widrig et al. ${ }^{32}$ propuseram comparar a diferença entre o ibuprofeno, AINES sintético, e a Arnica montana. Ressaltaram que haviam semelhança na atividade clínica ao ponto de nem pacientes nem médicos poderem distinguir entre os medicamentos.

\section{Uncaria Tomentosa}

A Uncaria tomentosa popularmente conhecida por "unha-de-gato", possui origem em áreas tropicais tal como América do Sul, américa central e na floresta amazônica2,33,34.

Os estudos para essa planta iniciaram em meados da década de 70, a mesma apresentou componentes importantes tal como: alcaloides, triterpenes, esteroides vegetais, glicosídeos e entre outros. Tendo assim a sua aplicação em abscessos, inflamações, febre, irregularidade menstrual, infecções bacterianas e fúngicas ${ }^{2,33}$.

Recomenda-se a utilização da casca do caule e da raiz, sendo assim: 0,5g em $150 \mathrm{~mL}$, via oral, somente em adultos. Dispõe de atividades anti-inflamatórias, em dores articulares e musculares agudas. Possui alguns efeitos colaterais tal como: cansaço, febre, diarreia, sintomas pancreáticos e alteração do nervo óptico 21,27 .

Paiva et al. $\frac{33}{1}$, em uma pesquisa para avaliar a eficácia da Uncaria tomentosa em relação ao Miconazol, observaram que este medicamento possui ação antifúngica e pode ser utilizado na Odontologia. Eles afirmaram então que clinicamente o Miconazol mostrou-se levemente superior a Uncaria tomentosa, laboratorialmente a mesma se tornou superior ao Miconazol. Com isso, revelou ser um excelente fitofármaco de uso antifúngico na Odontologia.

Em um outro momento, Allen-Hall et al. $\frac{35}{}$ garantiram que a Uncaria tomentosa possui uma resposta anti-inflamatória através da inibição do mecanismo da NF- $\beta$ que é um complexo proteico que desempenha funções tal como fator de transcrição, sendo assim promissora não somente como anti-inflamatório, como para formação de tumores evitando a metástase. 
Miller et al. ${ }^{34}$, apontaram que o medicamento fitoterápico POA-Phytolens (c) diz ser uma mistura entre a Uncaria Tomentosa e Phytolens (c) vendido como antioxidante, possui efeito sobre a inibição da atividade de COX-1 e COX-2. Porém, o POA-Phytolens (c) apresentou uma maior seleção na inibição do COX-2 do que alguns medicamentos como: ibuprofeno, naproxeno e indometacina.

Apesar da Uncaria tomentosa apresentar mecanismo de ação igual aos AINES comprovando assim a sua eficácia como medicamento fitoterápico, a literatura não discorre sobre possível existência de efeitos colaterais em pacientes renais crônicos e com comprometimento cardiovascular, devido a inibição de prostaglandinas. Este estudo comprova os menores efeitos colaterais destes medicamentos fitoterápicos quando comparados aos medicamentos sintéticos, apesar de terem, alguns deles, mecanismos de ação semelhantes aos AINES.

\section{Cúrcuma Longa}

A Cúrcuma longa, também conhecida como "cúrcuma", "açafrão", "açafroa", açafrão-da-terra", planta herbácea da família dos Zingiberaceae. Largamente produzida pela Índia, China e nos demais países asiáticos 27,36 .

Ghasemi, Bagheri e Barreto ${ }^{36}$ afirmaram que a cúrcuma vem sido utilizada há muitos séculos atrás, na medicina tradicional como um eficaz fármaco para curar e tratar lesões, distúrbios e várias outras doenças. Segundo Lüer et al. ${ }^{37}$, a Cúrcuma longa é apreciada por apresentar propriedades anti-inflamatórias e antimicrobianas, principalmente de forma tópica.

Na farmacopeia brasileira a Cúrcuma longa possui sinonímia de Cúrcuma doméstica, com orientações de uso 1 cápsula de 2 a $4 x$ ao dia, correspondendo a dose diária de 80 até $160 \mathrm{mg}$, quando a formulação for de álcool etílico a 96\% ${ }^{6}$. Na Cartilha do Conselho Regional de Farmácia do Estado de São Paulo afirma que a cúrcuma pode ser utilizada como tintura a $10 \%$ como modo de uso: $0,5-3 \mathrm{~mL}$ diluídos em $50 \mathrm{~mL}$ de água, $3 x$ ao diaㄹ․

Não há relato sobre os efeitos colaterais deste medicamento, porém os autores são unânimes quando afirmam a não utilização em junção com anticoagulantes, pois ela apresenta agentes anticoagulantes que podem desencadear um efeito aditivo sobre as plaquetas aumentando o risco à hemorragias ${ }^{6,27,38}$.

Marchi et al..$\underline{38}$ afirmaram que a cúrcuma longa apresenta uma ação de fotossensibilizante deixando os indivíduos susceptíveis a alterações cutâneas, além do uso prolongado desencadear úlceras gástricas.

Angeles, Hernandez e Martine $z^{39}$, atestaram que a cúrcuma é um fármaco de segurança com ação antiinflamatória, e beneficente em várias doenças tal como periodontite, estomatite, e mucosite oral. Lüer et al. ${ }^{37}$ afirmaram através de ensaios biológicos a eficácia da cúrcuma de forma tópica em mucosite oral, por meio de inibição do NF-k $\beta$. Lim et al. ${ }^{40}$ disseram que a aplicação tópica de cúrcuma melhora o processo de cicatrização de úlcera oral, e que a mesma pode ser utilizada como ferramenta médica eficaz e segura nesse tratamento.

Ghasemi, Bagheri, Barreto ${ }^{\frac{36}{6}}$ revelaram sobre os efeitos neuroprotetores, inibição de apoptose, lipoxigenase (LOX), inibidores de COX-2 e um mecanismo de resposta antioxidante. Abe, Hashimito e Horie ${ }^{41}$, verificaram que a Cúrcuma longa modula a ação celular de vários fatores de crescimento, citocinas e fatores de transcrição envolvidos no processo inflamatório, afirmando que a cúrcuma é capaz de inibir a produção de IL-8, IL-1 $\beta$ e fator de necrose tumoral (TNF-a).

Marchi et al. $\frac{38}{3}$ e Ghasemi, Bagheri e Barreto $\frac{36}{6}$ sustentaram que os efeitos descritos são antiinflamatório e efeito neuroprotetor. Já Angeles, Hernandez e Martinez $z^{39}$ afirmaram que a cúrcuma longa possui propriedades antiinflamatórias e analgésicas, além disso sustentaram que é uma alternativa terapêutica útil na pratica odontológica, considerada padrão ouro, que oferece uma alternativa para uso de AINES.

\section{Malva Sylvestris}

Malva sylvestris é denominada uma planta herbácea, conhecida como malva, malva branca e malva branca sedosa. Originada no continente Europeu, Africano, Asiático e Americano 2,42,43.

Para fins farmacológicos são utilizadas as folhas, flores e as raízes $²$ Segundo Aleluia et al. 2 , essa planta possui propriedades antiinflamatórias, antimicrobianas. Já Martins et al. $\underline{42}$ afirmaram que possui aplicação em casos de distúrbios gastrointestinal, doenças 
dermatológicas, dores menstruais, perturbações urológicas, doenças respiratórias e doenças orais. Seddighfar et al. ${ }^{44}$ disseram sobre a eficácia da Malva sylvestris na Odontologia em doenças inflamatórias como periodontite, gengivite e abcessos.

A malva pode ser utilizada por infusão $2 \mathrm{~g}$ em $150 \mathrm{~mL}$ de água ou $6 \mathrm{~g}$ em $150 \mathrm{~mL}$ de água. Utiliza $4 \mathrm{x}$ ao dia de forma oral e em forma tópica: fazer higienização com a infusão sobre o local afetado com algodão $3 x$ ao dia, ou fazer bochechos/gargarejos $3 x$ ao dia ${ }^{27}$.

Segundo Martins et al. ${ }^{42}$ em sua pesquisa afirmaram que a malva apresenta-se como um excelente antiinflamatório, agindo com seletividade em COX-2 e inibição de TXA2. Seddighfar et al. $\underline{44}$ disseram que as atividades anti-inflamatórias da Malva sylvestris se dar através da inibição das sínteses de prostaglandinas e mecanismos inibidores centrais, afirmando que este pode ser utilizado como medicamento para controlar dor e inflamação. Apesar da inibição de COX-2 efeitos colaterais na utilização da mesma não foram relatados pelos autores, mostrando assim vantagem sobre os AINES.

Sarmento et al..$_{5}^{45}$ pesquisaram sobre o potencial antimicrobiano dos antissépticos de uso popular e afirmaram que o Malvatricin ${ }^{\circledR}$ composto por malva, quinosol e tirotricina, mostrou bons resultados quando comparado a clorexidina a $2 \%$, principalmente sobre a C. tropicalis, afirmando o potencial antimicrobiano dos antissépticos. Benso et al. ${ }^{43}$ afirmaram o potencial antimicrobiano da Malva sylvestris, além da modulação de citocinas e receptores.

\section{Considerações finais}

Diante da resolução desta revisão de literatura, conclui-se que muitos medicamentos fitoterápicos possuem ação antiinflamatória, com mecanismo de ação semelhante aos AINES, sendo, portanto, uma alternativa terapêutica eficaz e menos custosa indicada para pacientes que possuem hipersensibilidades aos AINES, doenças renais crônicas, ou que são adeptos a tratamentos naturais. No entanto, observa-se que ainda não se conhece o perfil tóxico e risco para a saúde do ser humano de muitos fitoterápicos, sendo necessário mais estudos e que apesar de serem naturais os seus efeitos colaterais, a forma de administração e contra indicações devem ser de conhecimento do cirurgião-dentista para evitar complicações.

\section{Contribuição dos autores}

Moreira VL participou da concepção, realizou busca e revisão dos artigos científicos, além de ser responsável pela elaboração do manuscrito. Peixoto AP foi uma das idealizadoras do projeto, realizou o delineamento metodológico do artigo, e auxiliou na estruturação do manuscrito, direcionando o andamento do projeto, e participou da revisão final. Marchionni AMT foi um dos idealizadores do projeto, participou da definição do tema e revisão final do manuscrito.

\section{Conflitos de interesses}

Nenhum conflito financeiro, legal ou político envolvendo terceiros (governo, empresas e fundações privadas, etc.) foi declarado para nenhum aspecto do trabalho submetido (incluindo, mas não se limitando a subvenções e financiamentos, participação em conselho consultivo, desenho de estudo, preparação de manuscrito, análise estatística, etc.).

\section{Referências}

1. Francisco KSF. Fitoterapia: Uma opção para o tratamento
odontológico. Revista saúde. 2010;4(1):18-24.

2. Aleluia CM, Procópio VC, Oliveira MTG, Furtado PGS, Giovannini JFG, Mendonça SMS. Fitoterápicos na odontologia. Rev Odontol Univ Cid São Paulo. 2015; 27(2):126-34.

3. Santos RL, Guimarães GP, Nobre MSC, Portela AS. Análise sobre a fitoterapia como prática integrativa no Sistema Único de Saúde. Rev Bras PI Med. 2011; 13(4):486-491. http://dx.doi.org/10.1590/ S1516-05722011000400014

\begin{abstract}
4. Hasenclever L, Paranhos J, Costa CR, Cunha G, Vieira D. A indústria de fitoterápicos brasileira: desafios e oportunidades. Ciênc Saúde Coletiva. 2017; 22(8):2559-2569. http://dx.doi. org/10.1590/1413-81232017228.29422016
\end{abstract}

5. Monteiro MHDA, Fraga SAPM. Fitoterapia na odontologia: levantamento dos principais produtos de origem vegetal para saúde bucal. Revista Fitos. 2015;9(4):265-268. http://dx.doi. org/10.5935/2446-4775.20150021 
6. Agência Nacional de Vigilância Sanitária. Formulário de Fitoterápicos da Farmacopeia Brasileira/Agência Nacional de Vigilância Sanitária. Brasília: Anvisa, 2018.

7. Brasil. Resolução CFO-82/2008. Reconhece e regulamenta o uso pelo cirurgião-dentista de práticas integrativas e complementares à saúde bucal. Conselho Federal de Odontologia [Internet]. 2008 [acesso em 2019 out 28]. Disponível em: http://sistemas.cfo.org. br/visualizar/atos/RESOLU\%c3\%87\%c3\%830/SEC/2008/82

8. Korolkovas A, Ferreira El. Mecanismos gerais de ações dor fármacos. In: Silva P. Farmacologia. Rio de Janeiro: Guanabara Koogan; 2010. p. 98-109.

9. Monteiro ECA, Trindade JMF, Duarte ALBP, Chahade WH. Os antiinflamatórios não estoidais (AINEs). Temas Reumatol Clínica. 2008;9(2):53-63.

10. Melgaço SSC, Saraiva MIR, Lima TTC, Silva Júnior GB, Daher EF. Nefrotoxicidade dos anti-inflamatórios não esteroidais. Medicina. 2010;43(4):382-90. http://dx.doi.org/10.11606/issn.2176-7262. v43i4p382-390

11. Agência Nacional de Vigilância Sanitária. Paracetamol: riscos hepáticos relacionados ao uso do medicamento em combinação, em doses acima de 325mg [Internet]. 2014 [acesso em 2020 jul 04]. Disponível em: http://portal.anvisa.gov.br/

12. Silva JM, Mendonça PP, Partata AK. Anti-inflamatórios não-esteróides e suas propriedades gerais. Rev Cient ITPAC. 2014;7(4):1-15.

13. Lucas GNC, Leitão ACC, Alencar RL, Xavier RMF, Daher EF, Silva Junior GB. Pathophysiological aspects of nephropathy caused by non-steroidal anti-inflammatory drugs. J Bras Nefrol. 2019;41(1):124-30. http://dx.doi.org/10.1590/2175-8239jbn-2018-0107

14. Batlouni M. Anti-inflamatórios não esteroides: Efeitos cardiovasculares, cérebro-vasculares e renais. Arq Bras Cardiol. 2010;94(4):556-63. http://dx.doi.org/10.1590/50066$\underline{782 \times 2010000400019}$

15. Parolia A, Thomas MS, Kundabala M, Mohan M. Propolis and its potential uses in oral health. International Journal of Medicines and Medical Sciences. 2010;2(7):210-215.

16. Franchin M, Freires IA, Lazarini JG, Nani BD, Cunha MG, Colón DF et al. The use of Brazilian propolis for discovery and development of novel anti-inflammatory drugs. Eur J Med Chem. 2018;153:49-55. http://dx.doi.org/10.1016/j.ejmech.2017.06.050

17. Salehi M, Saeedi M, Ghorbani A, Ghodrati P, Moosazadeh M, Rostamkalaei S. et al. The Effect of Propolis Tablet on Oral Mucositis Caused by Chemotherapy. GMJ. 2018;29(3)196-201. http://dx.doi.org/10.12996/gmj.2018.55
18. Bolouri AJ, Pakfetrat A, Tonkaboni A, Aledavood SA, Najafi MF, Delavarian $Z$ et al. Preventing and Therapeutic Effect of Propolis in Radiotherapy Induced Mucositis of Head and Neck Cancers: A Triple-Blind, Randomized, Placebo-Controlled Trial. Iran J Cancer Prev. 2015;8(5):e4019. http://dx.doi.org/10.17795/ijcp-4019

19. Stähli A, Maheen CU, Strauss FJ, Eick S, Sculean A, Gruber R. Caffeic acid phenethyl ester protects against oxidative stress and dampens inflammation via heme oxygenase 1. Int J Oral Sci. 2019;11(1):1-8. http://dx.doi.org/10.1038/s41368-018-0039-5

20. Machado AC, Oliveira RC. Medicamentos fitoterápicos na odontologia: evidências e perspectivas sobre o uso da aroeirado-sertão (Myracroduon Urundueuva Allemão). Rev Bras Plantas Med. 2014;16(2):283-289. http://dx.doi.org/10.1590/S1516$\underline{05722014000200018}$

21. Ramos ACD, Machado MSL, Filho JAR, Freitas MCL, Silva ML, Pessoa PJB. Cartilha de Plantas Medicinais e Medicamentos Fitoterápicos [Internet]. 2014 [acesso em 2020 jan 11]. Disponível em: http://farmacia.saude.pe.gov.br/sites/farmacia.saude.pe.gov. br/files/cartilha.pdf

22. Soares DGS, Oliveira CB, Drumond MRS, Padilha WWN. Atividade Antibacteriana da Tintura de Aroeira (Schinus terebinthifolius) na descontaminação de escovas dentais contaminadas pelo S. mutans. Pesq Bras Odontoped Clin Intgr. 2007; 7(3):253-257. http://dx.doi.org/10.4034/1519.0501.2007.00 $\underline{73.0010}$

23. Machado AC, Sousa LP, Saldanha LL, Pieroni LG, Matos AA, Oliveira FA et al. "Aroeira" (Myracroduon Urundueuva) metanol extract: the relationship between chemical compounds and celular effects. Pharmaceutical Biology. 2016;54(11):2737-2741. http://dx.doi.org/10.1080/13880209.2016.1182555

24. Souza SMC, Aquino LCM, Milach Jr AC, Bandeira MAM, Nobre MEP, Viana GSB. Antiinflammatory and Antiulcer Properties of Tannins from Myracrodruon urundeuva Allemão (Anacardiaceae) in Rodents. Phytother Res. 2006;21(3):220-5. http://dx.doi. org/10.1002/ptr.2011

25. Quinelato V, Balduino A, Guimarães JP. Arnica Montana e desordens musculares mastigatórias. Rev Bras Odontol. 2011;68(2):225-228.

26. Iannitti T, Morales-Medina JC, Bellavite P, Rottigni V, Palmieri B. Effectiveness and safety of Arnica Montana in post-surgical setting, pain and inflammation. Am J Ther. 2016;23(1):184-97. http://dx.doi.org/10.1097/MJT.0000000000000036

27. Conselho Regional de Farmácia do Estado de São Paulo. Plantas Medicinais e fitoterápicos. 4a ed. [Internet]. 2019. Disponível em: http://www.crfsp.org.br/images/cartilhas/ PlantasMedicinais.pdf 
28. Berges C, Fuchs D, Opelz G, Daniel V, Naujokat C. Helenalin suppresses essential immune functions of activated CD4+ T cells by multiple mechanisms. Mol Immonol. 2009;46(2009):2892-2901. http://dx.doi.org/10.1016/j.molimm.2009.07.004

29. Tornhamre S, Schimidt TJ, Glaser BN, Ericsson I, Lindgren JA. Inhibitory effects of helenalin and related compounds on 5-lipoxygenase and leukotriene C4 synthase in human blood cells. Biochem Pharmacol. 2001;62(7):903-11. http://dx.doi.org/10.1016/ s0006-2952(01)00729-8

30. Verma N, Tripathi SK, Sahu D, Das HR, Das RH. Evaluation of inhibitory activities of plant extracts on production of LPS-stimulated pro-inflammatory mediators in 7774 murine macrophages. Mol Cell Biochem. 2010;336(1):127-35. http:// dx.doi.org/10.1007/s11010-009-0263-6

31. Jäger C, Hrenn A, Zwingmann J, Suter A, Merfort I. Phytomedicines prepared from Arnica flowers inhibit the transcription factors AP-1 and NF-B and modulate the activity of MMP1 and MMP13 in human and bovine chondrocytes. Planta Med. 2009; 75(12):1319-25. http://dx.doi. org/10.1055/s-0029-1185668

32. Widrig R, Suter A, Saller R, Melzer J. Choosing between NSAID and arnica for topical treatment of hand osteoarthritis in a randomised, double-blind study. Rheumatol Int. 2007;27(6):58591. http://dx.doi.org/10.1007/s00296-007-0304-y

33. Paiva LCA, Ribeiro RA, Pereira JV, Oliveira NMC. Avaliação clínica e laboratorial do gel da Uncaria tomentosa (Unha de Gato) sobre candidose oral. Rev Bras Farmacogn. 2009;19(2 A):4238. http://dx.doi.org/10.1590/S0102-695X2009000300015

34. Miller AK, Benson JM, Muanza DN, Smith JR, Shepherd DM. Anti-inflammatory effects of natural product formulations on murine dendritic cells. J Diet Suppl. 2011;8(1):19-33. http://dx.doi. org/10.3109/19390211.2010.542233

35. Allen-Hall L, Arnason JT, Cano P, Lafrenie RM. Uncaria tomentosa acts as a potent TNF-a inhibitor through NF-KB. J Ethnopharmacol. 2010;127(3):685-93. http://dx.doi.org/10.1016/j. jep.2009.12.004

36. Ghasemi F, Bagheri H, Barreto GE, Read MI, Sahebkar A. Effects of Curcumin on Microglial Cells. Neurotox Res. 2019;36(1):12-26. http://dx.doi.org/10.1007/s12640-019-00030-0
37. Lüer SC, Goette J, Troller R, Aebi C. Synthetic versus natural curcumin: Bioequivalence in an in vitro oral mucositis model. BMC Complement Altern Med. 2014;14:53. http://dx.doi. org/10.1186/1472-6882-14-53

38. Marchi JP, Tedesco L, Melo AC, Frasson AC, França VF, Satos SW et al. Curcuma longa I., o açafrão da terra, e seus benefícios medicinais. Arq Ciênc Saúde UNIPAR. 2016;20(3):189-94. http:// dx.doi.org/10.25110/arqsaude.v20i3.2016.5871

39. Ángeles CDM, Hernández EL, Hernández ALG, Martínez IOP. Curcumina, una alternativa terapéutica para la clínica dental (Parte I): antiinflamatorio y analgésico. Revista ADM. 2016;73(5):245-9.

40. Lim YS, Kwon SK, Park JH, Cho CG, Park SW, Kim WK. Enhanced mucosal healing with curcumin in animal oral ulcer model. Laryngoscope. 2016;126(2):68-73. http://dx.doi.org/10.1002/ lary. 25649

41. Abe $Y$, Hashimoto $S$, Horie T. Curcumin inhibition of inflammatory cytokine production by human peripheral blood monocytes and alveolar macrophages. Pharmacol Res. 1999;39(1):41-7. http://dx.doi.org/10.1006/phrs.1998.0404

42. Martins CAF, Campos ML, Irioda AC, Stremel DP, Trindade ACLB, Pontarolo R. Anti-Inflammatory Effect of Malva sylvestris, Sida cordifolia, and Pelargonium graveolens Is Related to Inhibition of Prostanoid Production. Molecules. 2017; 22(11):1-15. http://dx.doi.org/10.3390/molecules22111883

43. Benso B, Rosalen PL, Alencar SM, Murata RM. Malva sylvestris inhibits inflammatory response in oral human cells. An in vitro infection model. PLoS One. 2015;10(10):e0140331. http://dx.doi. org/10.1371/journal.pone.0140331

44. Seddighfar M, Mirghazanfari SM, Dadpay M. Analgesic and anti-inflammatory properties of hydroalcoholic extracts of Malva sylvestris, Carum carvi or Medicago sativa, and their combination in a rat model. J Integr Med. 2020;18(2):181-188. http://dx.doi. org/10.1016/j.joim.2020.02.003

45. Sarmento DJS, Monteiro BVB, Melo MCN, Lima KC. Potencial antimicrobiano dos antissépticos de uso popular anapyon ${ }^{\circledR}$, Água Rabelo $\circledast$ e Malvatricin $®$ sobre microrganismos do meio ambiente oral. Pesq Bras Odontoped Clin Integr. 2014;13(4):309-14. http:// dx.doi.org/10.4034/PBOCI.2013.134.02 